\title{
Diseño y Desarrollo de un Objeto Virtual de Aprendizaje para un Curso de Electrónica*
}

\section{Design and Development of a Learning Object for an Electronics Course}

\author{
DOI: http://dx.doi.org/10.17981/ingecuc.12.1.2016.01
}

Artículo de Investigación cientifica - Fecha de recepción: 06 de mayo de 2015 - Fecha de aceptación: 23 de noviembre de 2015.

\author{
Mónica Silva Quiceno \\ Magíster en Tecnología Educativa y Medios Innovadores para la Educación. \\ Universidad Cooperativa de Colombia. Villavicencio (Colombia). monica.silva@campusucc.edu.co \\ Piedad Chica Sosa \\ Especialista en Ingeniería de Software. Universidad Cooperativa de Colombia. Villavicencio (Colombia). \\ piedad.chica@campusucc.edu.co
}

Para citar este artículo / To reference this paper:

M. Silva Quiceno y P. Chica Sosa, "Diseño y desarrollo de un Objeto Virtual de Aprendizaje para un curso de Electrónica", INGE CUC, vol. 12, no. 1, pp. 9-20, 2016. DOI: http://dx.doi.org/10.17981/ingecuc.12.1.2016.01

\begin{abstract}
Resumen-- Este artículo presenta el diseño y desarrollo de un Objeto Virtual de Aprendizaje - OVA. El objetivo es lograr una herramienta computacional para facilitar la comprensión de los temas y permitir el acompañamiento durante el tiempo de trabajo independiente de los estudiantes del curso de Electrónica Básica del programa de Ingeniería de Sistemas de la Universidad Cooperativa de Colombia. Se utilizó la metodología de Material Educativo Computarizado-MEC de Galvis Panqueva que contempla las fases de análisis, diseño, desarrollo, evaluación e implantación. Los componentes pedagógicos se analizaron mediante la identificación de estilos de aprendizaje empleando el formato de encuesta de Felder y Silverman. Como resultado se obtuvo un OVA que permite el ingreso de tres tipos de usuario, definiendo para cada uno de ellos un rol específico y controlado mediante el inicio de sesión que habilita o deshabilita las opciones correspondientes a cada rol, contribuyendo al cumplimiento de las competencias del curso.
\end{abstract}

Palabras claves-- Acompañamiento; estilos de aprendizaje; material educativo computarizado; objeto virtual de aprendizaje; rol.

\begin{abstract}
This paper presents the design and development of a Virtual Learning Object. The aim is to achieve a computational teaching tool that facilitates the understanding of the course topics and allows the assistance of students during their independent study hours of the Basic Electronics course offered by the Computer Science program at the Universidad Cooperativa de Colombia. To develop this tool, the MEC (Computarized Educational Material) methodology proposed by Galvis Panqueva was used; this method includes the phases of analysis, design, development, testing and deployment. Pedagogical components were analyzed by identifying learning styles; for this, a survey of the Felder and Silverman type was conducted to a group of students. The Virtual Learning Object permits three types of user, for whom specific roles were defined and controlled by login features that enable or disable the options for each role, contributing to fulfill the tasks of the course.
\end{abstract}

Keywords-- Assistance; Computerized Educational Material; Virtual Learning Object; Learning Styles; Role.

\footnotetext{
* Artículo de investigación científica derivado del proyecto de investigación titulado: "Diseño y desarrollo de un Objeto Virtual de Aprendizaje para el curso de electrónica”. Financiado por el Consejo Nacional de Investigaciones de la Universidad Cooperativa de Colombia. Fecha de inicio: julio de 2011. Fecha de finalización: octubre 2013.
} 


\section{INTRODUCCIÓN}

Este artículo es producto de la realización de un proyecto para el curso de Electrónica Básica del programa de Ingeniería de Sistemas de la Universidad Cooperativa de Colombia, sede Villavicencio. Se tomaron como objeto de estudio a sus estudiantes y las actividades que se realizan en este curso para su aprobación y el cumplimiento de competencias. El proyecto se basa en la creación de un OVA que mejore la accesibilidad a los conocimientos por parte de los estudiantes, buscando que mediante estrategias de aprendizaje diferentes a la clase magistral, laboratorios o talleres, el estudiante pueda adquirir las competencias descritas en los programas del curso.

El punto de partida para el diseño del OVA es el resultado de estudios realizados en neurofisiología y psicología que muestran un nuevo enfoque sobre cómo los seres humanos aprenden y donde se pone en evidencia que no existe una sola forma de aprender, cada persona tiene una forma o estilo particular con la cual establece una relación con el mundo y, por lo tanto, para aprender [1]-[2]. Por esta razón, la implementación del OVA para el curso de Electrónica Básica busca ser esa estrategia de aprendizaje que cautive y facilite el entendimiento de los temas impartidos en el curso.

Actualmente, las estrategias utilizadas para el desarrollo del curso no se apartan de la clase magistral, es decir, se hace uso del tablero y escasamente recurren a prácticas en el laboratorio; asimismo, para valorar los conocimientos aprendidos se recurre a métodos tradicionales de evaluación, que en ocasiones generan apatía por parte de los estudiantes y los aleja de la posibilidad de obtener buenos resultados.

Por su parte, los OVA facilitan la interacción del estudiante con las temáticas del curso [3]. Su diseño e implementación busca motivar a los estudiantes al uso de las plataformas institucionales, a ser autodidactas y a medirse a través del uso la autoevaluación, permitiendo validar los conocimientos adquiridos y brindado una opción para el buen uso del tiempo libre.

\section{REVISIÓN LiterARIA}

Esta investigación responde a teorías relacionadas con los estilos de aprendizaje, el concepto de OVA y los enfoques y modelos pedagógicos pertinentes para el diseño de un OVA desde el punto de vista de los modelos que benefician la formación virtual, como el aprendizaje autónomo y el aprendizaje significativo [4]-[5]. Se hace una revisión literaria con el fin de presentar los conceptos relacionados con la aplicación de tecnologías en la educación y los trabajos realizados en esta área, así como el rol del docente en el uso de las tecnologías.

\section{A. Definición de TIC}

La ley 1341 de 2009 de la República de Colombia define principios y conceptos sobre la sociedad de la información y la organización de las Tecnologías de la Información y las Comunicaciones (TIC) y crea la Agencia Nacional del Espectro. Define las Tecnologías de la Información y las Comunicaciones, como el conjunto de recursos, herramientas, equipos, programas informáticos, aplicaciones, redes y medios que permiten la compilación, procesamiento, almacenamiento y transmisión de información, como: voz, datos, texto, video e imágenes [6].

Otra definición formal de Tecnologías de la Información y las Comunicaciones en el contexto colombiano es: "[1]as Tecnologías de la Información y las Comunicaciones, TIC, pueden definirse como el conjunto de instrumentos, herramientas o medios de comunicación, como la telefonía, los computadores, el correo electrónico y la Internet, que permiten comunicarse entre sí a las personas u organizaciones" [7].

\section{B. Las TIC en la educación}

En el contexto actual, las instituciones de educación se enfrentan a cambios planteados por la nueva sociedad de la información y el lugar que tienen en el conjunto del sistema de enseñanza, esto les implica involucrarse en procesos de calidad y ampliación de cobertura, considerando el uso de tecnología en la educación [8]-[9].

En términos generales, las principales funciones de las TIC en la educación están asociadas a las posibilidades de usarlas en este entorno como medio de expresión para generar documentos electrónicos, presentaciones, videos, imágenes [10].

Hay que tener claro que no basta con utilizar las TIC, se debe analizar si su incorporación realmente está produciendo un correcto aprendizaje. Este es uno de los elementos de la docencia que más preocupa a los profesores pues necesitan encontrar la forma de evaluar el aprendizaje que se ha producido gracias a la incorporación de las TIC. Los profesores no quieren un entorno tecnológico sino un servicio de apoyo en la universidad que en cualquier momento ayude a resolver problemas o mejorar el trabajo [11].

Múltiples trabajos ponen en evidencia esta realidad en el actual sistema de educación. El uso de tecnologías en la educación puede tener la finalidad de desarrollar las capacidades cognitivas del individuo; puede también tener el enfoque del trabajo colaborativo y en red para el desarrollo de la participación y de la ciudadanía, tal como se menciona en el libro del desafío de las TIC para el cambio educativo [12].

El trabajo "Análisis de las estrategias de enseñanza con tecnologías de la información, ¿un nuevo contexto metodológico de secundaria?", busca pro- 
fundizar en la relación que se va configurando entre la metodología didáctica existente en los centros de enseñanza secundaria y la utilización de las TIC [13].

El trabajo realizado por Washington Antonio Cevallos Gamboa, docente de la Facultad de Ingeniería Eléctrica y Computación de la Escuela Superior Politécnica del Litoral de Guayaquil, Ecuador, "Desarrollo de un marco referencial para la implantación, transferencia y evaluación de las TIC en las universidades”, logró poner en evidencia que no todas las instituciones de educación superior introducirán cambios institucionales mediatos para incursionar en las TIC. Las universidades en el país están dando pasos hacia la integración de las TIC, pero es necesario entender que éstos deben ir más rápido a pesar de la complejidad que esto pueda representar, si no de nada servirá entrar en el reto [14].

Otros autores como Salinas, plantea "modelos flexibles" como respuesta de las universidades a la sociedad de la información, argumenta la necesidad de adoptar modelos flexibles de enseñanza-aprendizaje y expone la necesidad de incorporar las TIC en la educación superior como respuesta de las instituciones de educación superior a los cambios de la sociedad actual [15].

Así mismo, Camargo, con el trabajo denominado "Incorporación de las TIC en el aula de matemáticas en la básica primaria del centro educativo Minas de Iracal, sede la Honda" tiene como objetivo promover el uso de las herramientas tecnológicas y materiales lúdicos en el proceso de enseñanza - aprendizaje de las matemáticas optimizando el rendimiento académico. El autor considera que con estas herramientas se atrae a los estudiantes a un entorno dinámico y más llamativo que permitirá disminuir los prejuicios que se tienen sobre la aparente dificultad de las matemáticas [16].

Por otra parte, el trabajo de incorporación de entornos tecnológicos de aprendizaje a la cultura escolar, proyecto de innovación educativa en matemáticas y ciencias en escuelas secundarias públicas de México, muestra una forma de enseñanza de la física y las matemáticas con tecnología. Este trabajo hoy sirve de referencia para el programa de reforma educativa en el sistema de educación secundaria en México [17].

La importancia adquirida por las TIC, y todo lo que su uso ha generado en el ámbito académico, ha hecho que profesores y estudiantes se vean obligados a asimilar gran cantidad de conocimiento en poco tiempo y a ritmos acelerados, de ahí surge la importancia de que las TIC sean incorporadas en los procesos de enseñanza - aprendizaje de cualquier programa de educación, pues éstas han permitido la evolución del proceso educativo y han contribuido en la forma en cómo el estudiante asimila contenidos y los usa para comprender y analizar situaciones de su entorno [18].

\section{Herramientas tecnológicas en la educación}

Las generaciones que proceden de los nuevos sistemas educativos y de una cultura audiovisual y tecnológica manifiestan nuevas formas de adquirir el conocimiento y, por tanto, de aprender. Cada vez resulta más difícil encontrar acciones formativas que no estén apoyadas en diferentes medios tecnológicos [19].

El uso de herramientas tecnológicas en ambientes académicos es más común cada día y los docentes descubren los beneficios de involucrarlos como apoyo a sus clases presenciales, sin embargo, en ocasiones la calidad de los materiales es pobre o el aprendizaje en entornos virtuales deriva demasiado trabajo para el docente [20]. No obstante, esto se puede evitar si se hace una evaluación seria de las herramientas tecnológicas empleadas y se establece una pauta para su uso y aplicación.

Dentro del esfuerzo de la comunidad académica, usar herramientas de carácter tecnológico ha sido una actividad permanente; el avance progresivo de las tecnologías habilita nuevas herramientas que pueden ser exploradas y usadas en escenarios de aprendizaje [21]. Por esta razón es necesario realizar una evaluación que identifique en qué casos es favorable su uso y cuáles son las indicadas en cada caso.

Se debe tener muy claro que el uso de las herramientas tecnológicas no puede relegar ni alterar el puesto que ocupan las instituciones dentro de la formación del estudiante, pues ellas son el pilar fundamental de los procesos académicos, tampoco se puede pretender que se usen indiscriminadamente sin tener en cuenta su adecuación didáctica.

\section{Rol del profesor}

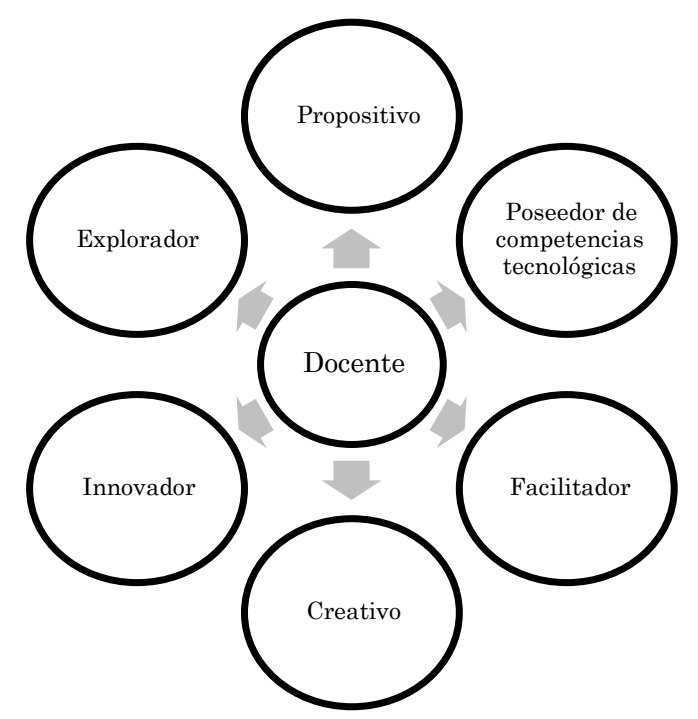

Fig. 1 . Características del docente. Fuente: Autores 
El rol del profesor actual debe responder de forma eficaz a los cambios y a las exigencias del entorno, modificando en cierta parte la forma cómo lleva a cabo el proceso de enseñanza - aprendizaje [22]. Por esto es necesario que el profesor asuma un rol más activo, propositivo y tenga un manejo básico de la tecnología, y que adicionalmente posea ciertas características que faciliten su trabajo con este tipo de herramientas en el aula, algunas de ellas se pueden observar en la Fig. 1.

El profesor puede participar en la formación de entornos de aprendizaje más participativos, flexibles y multidireccionales, ya que la información con el uso de herramientas tecnológicas se puede dar en ambos sentidos, resaltando que éstas son atractivas y permiten el acceso a múltiples entornos de aprendizaje, la personalización de los procesos de aprendizaje, una mayor flexibilidad en los estudios, la cercanía al docente, un mayor número de instrumentos para el proceso educativo, el compañerismo y la colaboración.

Dentro de las investigaciones relacionadas con esta temática se encuentran variedad de estudios con buenos resultados, de diferentes países y autores, que sirvieron para sustentar esta investigación. Trabajos como "Implicaciones pedagógicas y actitud del docente ante el uso de las TIC en el aula de ELE”, analizan la situación educativa actual con respecto a la integración de las tecnologías y las transformaciones pedagógicas consecuentes dentro del ámbito de la enseñanza de segundas lenguas y de ELE [23]. Teniendo en cuenta la evidente necesidad del uso de estas herramientas en los nuevos modelos de enseñanza, se plantea la posición del docente y su concienciación ante estos recursos.

El uso que se hace de las TIC en la docencia universitaria produce un mayor acercamiento a la profesión para la cual se les capacita, lo que permite la adquisición de competencias profesionales, además, se puede obtener una mayor atención personalizada y supervisión continua por parte de los profesores y se potencian competencias transversales de autonomía, responsabilidad, estudio continuado, aprendizaje colaborativo, etc. [24].

También, se observa cómo los profesores se capacitan utilizando estos recursos, trabajos como "Una manera de integrar las herramientas tecnológicas en la formación de los maestros" muestran cómo la innovación en la didáctica puede tener un impacto en el aprendizaje de estudiantes de Magisterio que cursan la asignatura Organización del centro escolar, donde a través de un entorno didáctico apoyado en herramientas tecnológicas se logra optimizar el aprendizaje, notando resultados positivos al finalizar el curso, pues hubo un aumento en la notas con respecto al curso anterior y una valoración positiva por parte de los estudiantes [25].

\section{E. Entornos Virtuales de Aprendizaje y Objetos Virtuales de Aprendizaje}

Los términos de referencia presentes en el desarrollo del proyecto y de la investigación hacen relación a la didáctica, el currículo educativo, el aprendizaje, el aprendizaje autónomo, el aprendizaje cooperativo y el aprendizaje significativo.

La didáctica hace referencia al conjunto de principios, criterios, normas, recursos y técnicas educativas propuestas con el fin de asegurar a los educadores los medios de una actuación segura, económica y eficaz sobre los aprendices [26].

El aprendizaje hace referencia al cambio más o menos permanente de conducta que se produce como resultado de la práctica [27].

El currículo educativo es una estructura que organiza la forma de construir un proceso educativo, para ello se centra en un propósito: ¿para qué enseñar?; una secuencia: ¿cuándo enseñarlo?; los recursos: ¿con qué enseñarlo?; el contenido: ¿qué enseñar?; una metodología: ¿cómo enseñarlo?; y evaluación: ¿se cumplió? [28].

Un Entorno Virtual de Aprendizaje se define como una plataforma tecnológica que trata de reproducir las condiciones y recursos educativos de una clase presencial y proporciona a profesores y estudiantes las facilidades para la comunicación y la interacción, venciendo de esta manera la necesidad de los actores implicados en el proceso de enseñanza - aprendizaje de coincidir temporal y geográficamente [29].

Es necesario generar, tanto desde el sector público como del privado, políticas para que la utilización de las tecnologías promueva una mejora en la calidad de la educación y amplíe la gama de oportunidades educativas para todos los sectores [30], pero sobre todo, sirve como instrumento para incorporar a la sociedad de la información los estratos de menores recursos que están quedando rezagados en la distribución del conocimiento (exclusión digital) [31].

Para contribuir al mejoramiento de esta situación, se viene trabajando ampliamente en el desarrollo de OVA, los cuales están definidos por el Ministerio de Educación colombiano como: "todo material estructurado de una forma significativa, asociado a un propósito educativo y que corresponda a un recurso de carácter digital que pueda ser distribuido y consultado a través de la Internet" [32].

Para que se constituyan en facilitadores del aprendizaje, los OVA deben concebirse como unidades mínimas de contenido didáctico con sentido en sí mismos que sirvan de anclaje para aprendizajes posteriores [33]. De igual forma, deben contener elementos altamente motivadores e interactivos haciendo uso de la narrativa hipermedial para que establezca relaciones que complementen la información a través de enlaces y mapas conceptuales que presenten la información de una manera sintética y estructurada, priorizando la internavegabilidad [34]. 


\section{F. Estilos de aprendizaje}

El estilo de aprendizaje puede concebirse como un planteamiento cognitivista, referido a la heurística del pensamiento y al conjunto de hábitos y formas de cada persona para pensar y actuar de acuerdo con determinada situación, cómo tiende a tomar decisiones en los diferentes entornos bajo condiciones de incertidumbre, ansiedad, presión, etc. [35].

En forma sencilla, se trata de cómo la mente procesa información, del modo como se vale de ciertas estrategias para trabajarla, (mapas mentales, mapas conceptuales, uve heurística, entre otras) o cómo esta información es influida por las representaciones internas de cada persona con la finalidad de lograr aprendizajes significativos [36].

Se ha logrado agrupar los estilos de aprendizaje y se han logrado encontrar una serie de características que son importantes de reconocer en la labor docente para la fase de planeación de las sesiones pedagógicas. Conforme un estudiante avance en su proceso de aprendizaje, descubre nuevas o mejores formas de aprender, por lo tanto, puede variar su estilo; además, esta situación dependerá de los contextos, tiempos de aprendizaje, resultados esperados, criterios de evaluación, etc. [37].

El estilo de aprendizaje de cada persona, según la escuela de programación neurolingüística (PNL), es la manera preferida que cada uno tiene de captar, recordar, imaginar o enseñar un contenido determinado [38].

Para la PNL, el canal perceptual por donde nos apropiamos de los contenidos (el ojo, el oído o el cuerpo en general) resulta fundamental en nuestras preferencias de aprendizaje. La mayoría de personas utilizan estos canales en forma desigual, potenciando unos y desatendiendo otros [39].

\section{Metodología}

Para el desarrollo del OVA se utilizó el modelo de MEC (materiales educativos computarizados) propuestoa por Galvis Panqueva, que hace referencia a la ingeniería de software educativo que conserva los pasos de: análisis, diseño, desarrollo, implementación y evaluación con el propósito de hacer un buen uso del material educativo cuando se requiera y adecuarlo a la necesidad respectiva [40]. El OVA se llevó a cabo en Flex, PHP y Flash.

\section{A. Modelo de Material educativo computarizado, MEC}

Material educativo computarizado o MEC es la denominación otorgada a las diferentes aplicaciones informáticas cuyo objetivo final es apoyar el aprendizaje. Se caracterizan porque es el estu- diante quien controla el ritmo de aprendizaje, la cantidad de ejercicios, decide cuándo abandonar y reiniciar, interactuar reiteradas veces, en fin, son muchos los beneficios. Por su parte, el profesor encuentra en ellos una ayuda significativa, pues en muchos casos toda la actividad del estudiante se registra en los MEC [41]. Estos emplean variedad de ayudas como se muestra en la Fig. 2.

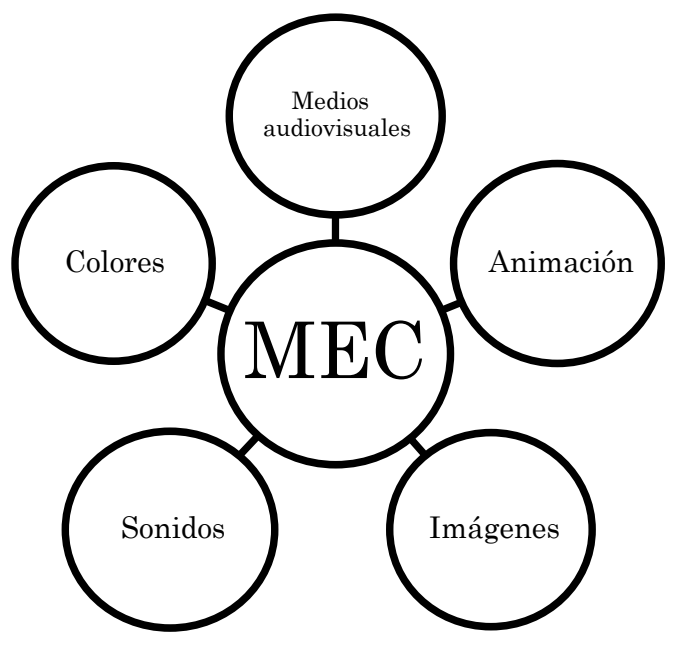

Fig. 2. MEC.

Fuente: Autores.

De igual forma, Galvis Panqueva categoriza las diferentes aplicaciones informáticas de acuerdo con el objetivo que buscan, el momento educativo en que se vayan a utilizar o la complejidad en el diseño de los mismos. Llegar a este tipo de productos, requiere de una revisión y reflexión teórica para acompañar la creación de este nuevo ambiente de aprendizaje.

Es indispensable reconocer las metodologías de desarrollo de software existentes para poder seleccionar la más adecuada. Anteriormente, existían métodos como la Enseñanza Asistida por Ordenador que nació en los años 60 en los Estados Unidos y heredó directamente los métodos de trabajo de la enseñanza programada propuestos y desarrollados por el psicólogo norteamericano Skinner a finales de los años 50; este planteamiento inicial basado en el neoconductismo consistía en usar máquinas de enseñar de encadenamiento lineal pregunta-respuesta-estímulo [42].

Para considerar que un MEC está diseñando correctamente, se debe tener en cuenta que garantice lo siguiente: facilitar la motivación, recordar el aprendizaje anterior, proporcionar nuevos estímulos, activar la respuesta de los alumnos, proporcionar información, estimular la práctica, establecer una secuencia de aprendizaje, propiciar recursos, generar efectos visuales y auditivos, ser cómodamente interactivos, poder procesar símbolos y ser modificables [43]. 


\section{1) Fase 1: Análisis y obtención}

En esta fase se identificaron las necesidades educativas reales que se requiere atender con el OVA a ser desarrollado. Se empleó el modelo de Felder y Silverman, el cual fue seleccionado por la aceptación que tiene entre los desarrolladores de sistemas de aprendizaje electrónico y porque el instrumento empleado para identificar el estilo de aprendizaje del estudiante ha sido validado [44]. El modelo de Ferlder y Silverman clasifica los estilos de aprendizaje a partir de cinco dimensiones: relativa al tipo de información, relativa al tipo de estímulos preferenciales, relativa a la forma de organizar la información, relativa a la forma de trabajar con la información y relativa a la forma de procesar y comprender la información.

A partir de estos planteamientos, Richard Felder desarrolla un instrumento para el Inventario de estilos de aprendizaje, diseñado a partir de cuatro escalas bipolares relacionadas con las preferencias para los estilos de aprendizaje, que son activo-reflexivo, sensorial-intuitivo, visual-verbal y secuencial-global. El instrumento consta de 44 ítems y ha sido utilizado para describir la relación de los estilos de aprendizaje con las preferencias de los estudiantes vinculando los elementos de motivación en el rendimiento académico [45].

Este instrumento fue aplicado a 36 estudiantes del programa de Ingeniería de Sistemas de V semestre que es donde está ubicado el curso y a estudiantes de IV semestre del mismo programa que serán los usuarios potenciales del mismo. La finalidad de la aplicación de este instrumento es identificar qué estilo de aprendizaje predomina en ellos y así revisar las vías más adecuadas para facilitar el proceso de aprendizaje para emplear estas características en el desarrollo del OVA. Los resultados obtenidos se muestran en las Tabla I, II, III y IV.

A partir de los resultados obtenidos se pudo determinar que el grupo de estudiantes del programa de Ingeniería de Sistemas encuestado tuvo predominancia por la opción A, lo cual significa que el grupo es activo, sensorial, visual y secuencial, lo cual es propicio para el desarrollo del OVA de electrónica.

Adicionalmente, se analizaron los contenidos y los elementos que intervienen en el curso de electrónica, como son las problemáticas y factores asociados al aprendizaje y comprensión de los temas, además de revisar las variables implicadas en el mismo, como: los modelos pedagógicos utilizados, la pedagogía del profesor, los factores asociados a la monotonía, la dificultad de los conceptos, el tipo de material, las dificultades en los laboratorios, la actualización de los recursos, las estrategias de comunicación, la motivación del profesor y de los participantes, las aptitudes de los estudiantes en el ingreso, la ubicación de habilidades al ingreso y durante el desarrollo del curso, entre otras.
TAbla I. Registro de Resultados de las PREguntas CORRESPONDIENTES A ACTIVO-REFLEXIVO

\begin{tabular}{|c|c|c|}
\hline \multirow{2}{*}{ Nro. Preg } & Act & Ref \\
\cline { 2 - 3 } & A & B \\
\hline 1 & 33 & 17 \\
\hline 5 & 19 & 15 \\
\hline 9 & 21 & 12 \\
\hline 13 & 24 & 23 \\
\hline 17 & 13 & 16 \\
\hline 21 & 20 & 21 \\
\hline 25 & 15 & 24 \\
\hline 29 & 25 & 16 \\
\hline 33 & 12 & 14 \\
\hline 37 & 20 & 52 \\
\hline 41 & & 524 \\
\hline
\end{tabular}

Fuente: Autores.

Tabla II. Registro de Resultados de las preguntas CORRESPONDIENTES A SENSORIAL-INTUITIVO

\begin{tabular}{|c|c|c|}
\hline \multirow{2}{*}{ Nro. Preg } & Act & Ref \\
\hline & $\mathrm{A}$ & $\mathrm{B}$ \\
\hline 2 & 21 & 15 \\
\hline 6 & 30 & 6 \\
\hline 10 & 23 & 13 \\
\hline 14 & 16 & 20 \\
\hline 18 & 28 & 8 \\
\hline 22 & 21 & 15 \\
\hline 26 & 19 & 17 \\
\hline 30 & 21 & 15 \\
\hline 34 & 15 & 21 \\
\hline 38 & 24 & 12 \\
\hline 42 & 25 & 11 \\
\hline \multirow{2}{*}{ Total Columna } & $\mathrm{A}$ & B \\
\hline & 243 & 153 \\
\hline Resta Menor al mayor & \multicolumn{2}{|c|}{90} \\
\hline Asignar Letra mayor & \multicolumn{2}{|c|}{$90 \mathrm{~A}$} \\
\hline
\end{tabular}

Fuente: Autores. 
Tabla III. Registro de Resultados de las PREguntas CORRESPONDIENTES A SECUENCIAL-GLOBAL

\begin{tabular}{|c|c|c|}
\hline \multirow{2}{*}{ Nro. Preg } & Act & Ref \\
\hline & A & B \\
\hline 4 & 20 & 16 \\
\hline 8 & 22 & 14 \\
\hline 12 & 20 & 16 \\
\hline 16 & 27 & 9 \\
\hline 20 & 28 & 8 \\
\hline 24 & 22 & 14 \\
\hline 28 & 14 & 22 \\
\hline 32 & 19 & 17 \\
\hline 36 & 16 & 20 \\
\hline 40 & 22 & 14 \\
\hline 44 & 21 & 15 \\
\hline \multirow{2}{*}{ Total Columna } & A & B \\
\hline & 231 & 165 \\
\hline Resta Menor al mayor & \multicolumn{2}{|c|}{66} \\
\hline Asignar Letra mayor & \multicolumn{2}{|c|}{$66 \mathrm{~A}$} \\
\hline
\end{tabular}

Fuente: Autores.

Tabla IV. Registro Resultados de las preguntas CORRESPONDIENTES A VISUAL-VERBAL

\begin{tabular}{|c|c|c|}
\hline \multirow{2}{*}{ Nro. Preg } & Act & Ref \\
\cline { 2 - 3 } & A & B \\
\hline 2 & 21 & 6 \\
\hline 6 & 30 & 13 \\
\hline 10 & 23 & 20 \\
\hline 14 & 16 & 8 \\
\hline 18 & 28 & 15 \\
\hline 22 & 21 & 15 \\
\hline 26 & 19 & 21 \\
\hline 30 & 21 & 12 \\
\hline 34 & 15 & 11 \\
\hline 38 & 24 & 90 \\
\hline 42 & 25 & \\
\hline
\end{tabular}

Fuente: Autores.

\section{2) Fase 2: Diseño}

Los hallazgos y recopilaciones de la fase anterior permitieron definir el diseño de forma directa. Con los resultados del análisis, se procedió a la orientación y se observó que el contenido del OVA se deriva de la necesidad o problema que justifica el mismo, así como de lo que se supone que un usuario del OVA ya sabe sobre el tema.

Entre los elementos a tener en cuenta, se establecen momentos para el diseño del OVA, los cuales son características de las necesidades y los propósitos, en este caso del curso de Electrónica; estos se describen a continuación:

- Entorno de diseño. En esta etapa del proyecto se diseñaron las condiciones y los requerimientos para laboratorios, simulaciones, videos, contenidos del material educativo, pruebas, evaluaciones para los estudiantes, motor de evaluación para el docente teniendo en cuenta los tipos de usuario.

- Diseño educativo. Se resolvieron los interrogantes que se refieren en el alcance de la propuesta, los contenidos, las estrategias, los apoyos pedagógicos y el tratamiento de los recursos que debía ser capaz de apoyar el OVA para responder en los propósitos del curso desde los componentes teóricos y prácticos.

- Diseño computacional. Se acude a las necesidades; se establecieron qué funciones serían deseables que cumpliera el OVA para el apoyo de sus usuarios, el profesor y los estudiantes, como se ilustra en el caso de uso mostrado en la Fig. 3. Esta propuesta busca que el OVA diseñado pueda brindar al estudiante la posibilidad de controlar la secuencia, el ritmo, la cantidad de ejercicios y de abandonar y reiniciar. Por otra parte, se piensa en este momento de la metodología ¿qué puede ofrecer el OVA al profesor? teniendo en cuenta aspectos como la posibilidad de editar los ejercicios o las explicaciones, llevar registro de los estudiantes que utilizan el material y del rendimiento que demuestran y hacer análisis estadísticos sobre variables de interés, entre otros.

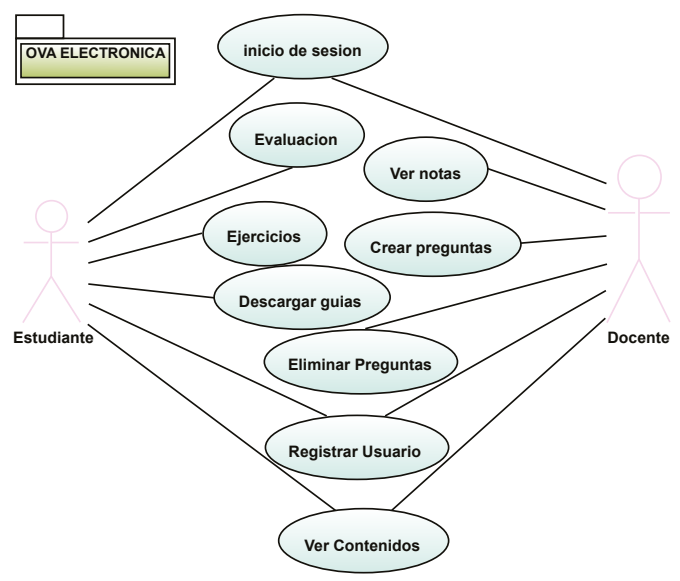

Fig. 3. Diagrama de caso de uso del OVA. Fuente: Autores. 
- Diseño de la comunicación. Se estableció la interacción hombre-máquina. Para especificarla, se determinó cómo se realizaría la comunicación del usuario con el OVA, estableciendo los dispositivos y códigos o mensajes (interfaz de entrada); también se hizo necesario establecer cómo se comunicaría el programa con el usuario, mediante qué dispositivos y valiéndose de qué códigos o mensajes (interfaz de salida) (Fig.4).

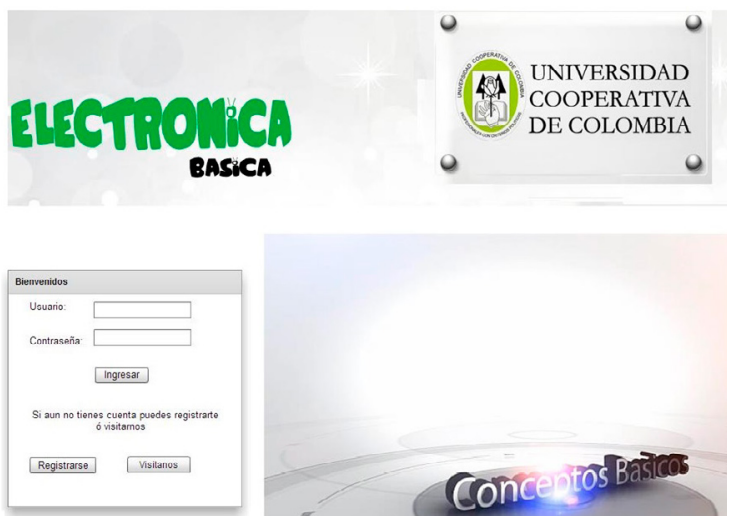

Fig. 4. Interfaz del OVA.

Fuente: Autores

- Preparación y revisión de los elementos del $O V A$. Es la fase final de la metodología en la etapa del diseño. Aquí se pretende llevar al terreno de la aplicación aquello que se ha concebido para el curso de Electrónica, y verificar las estrategias creadas frente a las necesidades y población a la que se dirige el mismo.

La forma más apropiada y la elegida en este caso para elaborar la aplicación se basa en bocetos en papel de cada uno de los ambientes que se van utilizar; otra forma complementaria es hacer lo equivalente pero en el computador. En esta parte intervienen expertos externos a los diseñadores, así como usuarios representativos de la población objeto. A cada uno de ellos debe someterse a consideración el bosquejo, conocer su reacción general y sus sugerencias particulares como base para realizar los ajustes al diseño donde sea pertinente.

\section{3) Fase 3: Desarrollo}

Según esta metodología, se contempla que una vez que se dispone de un diseño debidamente documentado, es posible llevar a cabo su implementación en el computador usando herramientas de trabajo que permitan al recurso humano asignado cumplir con las metas en términos de tiempo y de calidad del OVA. En este caso se acomodan los recursos y se realizan las disposiciones pertinentes para el caso.

El desarrollo fue ejecutado por los responsables del proyecto pero contó con el apoyo de otros miembros del grupo (director, codirector, profesores, pedagogos, asesores externos), lo que facilitó la retroalimentación de los diseños, permitiendo de esta forma consultar sobre la calidad de lo que se va haciendo y sobre detalles que surgieron a lo largo de la programación.

En esta fase fue importante la intervención de expertos en diseño gráfico para proveer los elementos que integraran el OVA, como una interfaz adecuada que motivara al estudiante a aprender, además, se hizo necesario integrarlos en un archivo HTML, generando aquí una plantilla con datos como la información general de la institución, el logo de la universidad, entre otros, con el fin de ofrecer a los estudiantes contenidos con un formato uniforme (Fig. 5).

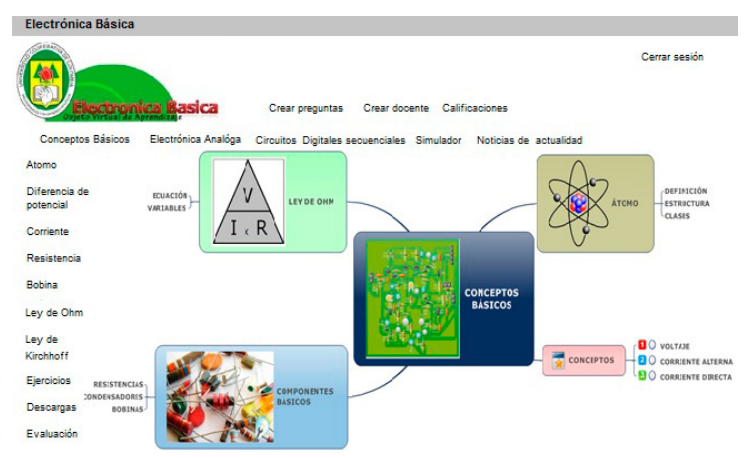

Fig. 5. Interfaz principal - navegación por módulos. Fuente: Autores

La Fig. 5 demuestra cómo se benefician los estilos de aprendizaje identificados (activo, sensorial, visual, secuencial) por cuanto implica una exploración en la pantalla para poder reconocer diferentes conceptos relacionados con el curso. El acceso a cualquiera de las unidades de aprendizaje despliega un menú en la parte izquierda de la pantalla dando la posibilidad de seleccionar alternativas alrededor del tema trabajado como son conceptos básicos, ejercicios, descargas, autoevaluación, entre otros.

Finalmente, en esta fase se hizo necesario un software generador de paquetes SCORM que permitiera crear y editar el metadato del OVA; aquí se seleccionó el que mejor se adaptaba a las necesidades y se procedió a ubicarlo en un repositorio temporal, lo que facilitó la labor de búsqueda y uso para efectos de la evaluación.

En cada unidad de aprendizaje se tiene acceso en forma libre a la autoevaluación, como se muestra en la Fig. 6. 


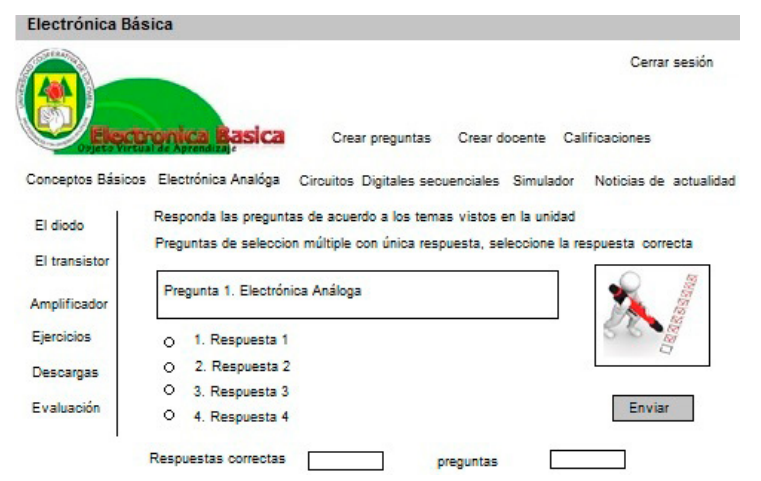

Fig. 6. Autoevaluación del OVA. Fuente: Autores

\section{4) Fase 4: Implementación}

Esta fase permite adoptar estándares para ser utilizados en diferentes plataformas, además provee un MEC que funciona sin la necesidad de estar conectado a Internet, facilitando su uso y reuso. Para que esto se dé de manera adecuada, se deben tener en cuenta las tecnologías a utilizar, como son los repositorios de OVA y los Sistemas de Gestión de Aprendizaje. El OVA se implementa en una plataforma institucional de e-learning denominada $\mathrm{Au}$ las extendidas.

Finalmente, se logró realizar una implementación en los lenguajes de programación Flex, PHP y Flash enfocando el desarrollo en el modelo vista controlador, permitiendo así que cada uno de los lenguajes esté presente en una parte especificada del aplicativo [46].

\section{Flex}

Adobe Flex es un framework de desarrollo de aplicaciones web interactivas: Aplicaciones Ricas de Internet, RIA. Se basa en la plataforma Flash combinando el lenguaje de marcas MXML y el lenguaje de programación Actionscript. Su entorno de desarrollo es Flex Builder [47]. Flex se encarga de realizar el módulo de vista, interfaces y consultas a cada una de las unidades mostrando los contenidos que se encuentran en cada una de ellas, además, se encarga de la integración entre PHP y Flash.

\section{Flash}

El software Adobe Flash Professional es un potente entorno de creación de animaciones y contenido interactivo y expresivo líder en el sector. Con éste se pueden diseñar experiencias interactivas envolventes que se presenten de forma uniforme en computadores de escritorio y múltiples dispositivos, incluidos tabletas, teléfonos inteligentes y televisores [48].

\section{PHP}

Es un lenguaje de código abierto muy popular, especialmente adecuado para desarrollo web y que puede ser incrustado en HTML. La mayor parte de su sintaxis ha sido tomada de C, Java y Perl con algunas características específicas de sí mismo. La meta del lenguaje es permitir rápidamente a los desarrolladores la generación dinámica de páginas [49].

\section{$M y S Q L$}

Es un sistema de gestión de bases de datos relacional. Fue creada por la empresa sueca MySQL AB, la cual tiene el copyright del código fuente del servidor SQL, así como también de la marca. MySQL es un software de código abierto, licenciado bajo la GPL de la GNU, aunque MySQL AB distribuye una versión comercial, en lo único que se diferencia de la versión libre es en el soporte técnico que ofrece y la posibilidad de integrar este gestor en un software propietario, ya que de otra manera, se vulneraría la licencia GPL [50].

Con el lenguaje interpretado PHP se realizan las operaciones de control requeridas para el funcionamiento del sistema, implementando las consultas necesarias a la base de datos y realizando la conexión entre la misma y el aplicativo; esa integración es gestionada con el motor de bases de datos MySQL, en donde se almacena la información del sistema.

Por último, todas las animaciones que se encuentran en el aplicativo están desarrolladas en Flash y su lenguaje de programación propio Actionscript 3.0 y Actionscript 2.0.

\section{5) Fase 5: Evaluación}

Esta fase busca contribuir a la depuración del OVA, teniendo claridad de que no se evaluará de manera individual cada objetivo de aprendizaje sino más bien se tomará el curso como un todo y teniendo en cuenta algunos criterios de referencia. La evaluación inicia con una prueba piloto de su utilización en una muestra representativa de los estudiantes del curso de Electrónica de V semestre del programa de Ingeniería de Sistemas de la Universidad Cooperativa de Colombia, sede Villavicencio, para los que se hizo la evaluación formativa. Los estudiantes manifestaron estar a gusto con el diseño del OVA y las diferentes opciones que presenta el menú.

En este punto, el OVA se encuentra listo para ser utilizado, razón por la cual es necesario que se diseñen pruebas sencillas de rendimiento, como listas de observación y cotejo, para determinar cuánto aprendieron los usuarios. Las pruebas se aplicarán al menos al inicio y al final del uso del material para contrastar las diferencias. Además 
de las pruebas de rendimiento es importante, para ver los propósitos del curso y los logros de los estudiantes de Electrónica, registrar cuánto tiempo se interactuó con el material para conocer el tiempo mínimo, máximo y promedio que se requiere para estudiar el OVA y sacarle provecho a la evaluación de la herramienta.

Para lograr condiciones normales, la prueba debe hacerse bajo circunstancias lo más cercanas a las del uso del material: en el momento de estudio debido y con los recursos y limitaciones del OVA que han sido previstos. Para obtener una muestra representativa conviene hacer una selección de individuos al azar entre la población objeto.

El proyecto de investigación se socializó en varios escenarios como:

- I Encuentro de Investigadores realizado en la Universidad Cooperativa de Colombia, sede Villavicencio, en el mes de noviembre de 2012, donde participaron estudiantes y docentes de la Universidad.

- Visita de estudiantes de la Universidad Distrital con el fin de conocer los proyectos de investigación del programa de Ingeniería de Sistemas en diciembre de 2012.

- Comité de investigaciones, donde se realizaron algunas observaciones en cuanto al OVA de Electrónica, las cuales fueron acatadas.

- Reunión con el grupo de ingenieros electrónicos de la Facultad en mayo de 2013, con el fin de conocer su opinión sobre el diseño del OVA. Los ingenieros electrónicos avalaron el diseño del OVA y reconocieron su importancia para el desarrollo del curso.

- Presentación de ponencia en el XX Congreso Internacional sobre educación Bimodal TELEDU 2014, en la ciudad de Medellín.

\section{Resultados y Discusiones}

- La aplicación del instrumento de Felder y Silverman permitió identificar las preferencias de los estudiantes en cuanto a estilos de aprendizaje, encontrándonos con un grupo activo, sensorial, visual y secuencial.

- Para el desarrollo del OVA se tuvieron en cuenta cuatro capítulos: conceptos básicos, diodos, circuitos digitales y circuitos digitales secuenciales, en cada uno de ellos se presentan los conceptos principales de una manera dinámica y se brinda la posibilidad de que el estudiante revise enlaces con videos, ejercicios y conceptos del tema tratado.

- El OVA para el curso de Electrónica Básica del programa de Ingeniería de Sistemas está desarrollado en un entorno web, buscando la implementación de la universidad virtual en el acompañamiento del trabajo independiente del estudiante, realizando los siguientes procesos:
- Introducción

- Inicio de sesión

- Registro de usuarios nuevos

- Desarrollo de temática

- Descarga de talleres

- Ejercicios desarrollados

- Actividades evaluativas

- Creación de preguntas para la evaluación

- Eliminación de preguntas

- Revisión de notas

- El OVA para el curso de Electrónica Básica permite el ingreso de tres tipos de usuario, definiendo para ellos tres roles específicos:

- Rol de administrador o docente: puede visualizar cada uno de los módulos del aplicativo, administrar las preguntas para las actividades evaluativas, obtener las calificaciones de los estudiantes tanto por modulo individual o una nota total del curso, además, a este rol se le permite registrar usuarios con los mismos permisos.

- Rol de estudiante: posee permisos básicos sobre el OVA y presenta la posibilidad de visualizar cada uno de los módulos del aplicativo, desarrollar las actividades evaluativas y navegar por todo el entorno Web, con lo cual podrá fortalecer los conocimientos adquiridos en clase para así aprobar satisfactoriamente el curso.

- Rol de visitante: este rol sólo permite visualizar los contenidos del OVA, dando la posibilidad de adquirir los conocimientos teórico-prácticos proporcionados; puede realizar los ejercicios propuestos pero no la evaluación de cada módulo. No se tienen en cuenta sus calificaciones.

Los permisos en el aplicativo se controlan mediante el inicio de sesión. Durante este proceso se lee el rol de cada usuario y de esta forma se habilita o deshabilitan las opciones correspondientes a cada rol.

- El OVA presenta la opción de autoevaluación, lo cual permite que el estudiante valide los conocimientos adquiridos a través de una serie de preguntas que son respondidas mediante la opción de selección múltiple, permitiendo que tome conciencia del proceso autónomo que tiene dentro del aprendizaje.

- El desarrollo del OVA para el curso de Electrónica Básica es una herramienta adicional con la que contarán los estudiantes del curso, lo cual brindará otra opción para fortalecer el aprendizaje y cumplir las competencias del curso y servirá de ejemplo para la creación de nuevos OVA en distintas áreas del conocimiento.

- A partir de la metodología empleada se logra un OVA dinámico que permitirá que semestre a semestre los profesores tengan la opción de ir alimentando la base de datos para variar el tipo de preguntas que se incluyan en la sección de evaluación. 


\section{Conclusiones}

- En la actualidad los OVA son de suma importancia en los modelos pedagógicos y en los programas por competencias, ya que permiten una interacción entre profesor y estudiante para el fortalecimiento del proceso enseñanza-aprendizaje y la aplicación de los conocimientos de la clase magistral por parte del estudiante para el logro de las competencias del curso.

- Los lenguajes de programación escogidos para el desarrollo del OVA cumplieron con las expectativas trazadas en la etapa de análisis y diseño, generando entre los tres lenguajes de programación usados (Flex, PHP, Flash-Actionscript 3.0) una combinación capaz de suplir los requerimientos funcionales establecidos.

- Desde el momento en que se implemente el uso del OVA se estará beneficiando tanto a los estudiantes del curso de Electrónica Básica como al profesor que dirige el curso. Aproximadamente cada año se beneficiará un total de 20 estudiantes del programa de Ingeniería de Sistemas, se busca que con el tiempo se pueda ampliar la cantidad de estudiantes beneficiados incorporando OVA en otros cursos del plan de estudios.

- Las ventajas de utilizar OVA en el aprendizaje de los temas del curso de Electrónica Básica radican en que el docente facilitador se encuentra de forma asincrónica - sincrónica con su estudiante en Ambientes Virtuales de Aprendizaje, por lo que el OVA se comporta como una extensión del docente, del conocimiento y de los aprendizajes que el estudiante debe adquirir: esa es su principal ventaja pedagógica, aparte de generar empatía, interés, animo, autoestima y autoaprendizaje.

- El uso del OVA permitirá al estudiante la selección de alternativas, como son conceptos básicos, ejercicios, descargas, autoevaluación, entre otros, con las cuales podrá reforzar los temas vistos en clase. Estas alternativas fueron implementadas gracias a la identificación de los estilos de aprendizaje de los estudiantes.

- Es necesario que antes de dar inicio al uso del OVA se realice una prueba del rendimiento al estudiante, la cual se vuelva a repetir al finalizar el semestre con el fin de comparar los resultados obtenidos gracias al uso del OVA.

\section{REFERENCIAS}

[1] J. Gómez, Neurociencia cognitiva y educación. Ciudad Universitaria de Lambayeque. Perú: Fondo Editorial FACHSE, 2004, pp. 160-176.

[2] A. Cisneros, Manual de estilos de aprendizaje. México: Secretaría de Educación Pública, 2004. [En línea]. Disponible en: http://estudiaen.jalisco.gob.mx/cepse/sites/ estudiaen.jalisco.gob.mx.cepse/files/cisneros_a._2004_ manual_estilos_de_aprendizaje.pdf
[3] L. Fajardo, M. Sotelo y F. Moreno. El uso de los OVAS como estrategia de enseñanza - aprendizaje bajo un esquema de educación bimodal. [En línea]. Disponible en: http:// www.konradlorenz.edu.co/images/pdf/2012_07_26_ponencia_teledu_texto.pdf.

[4] C. Sierra, Educación virtual aprendizaje autónomo y construcción de conocimiento. Bogotá, Colombia: Editorial Politécnico Grancolombiano, 2012, pp. 23-35.

[5] M. Moreira, Aprendizaje significativo: un concepto subyacente. Presentado el Encuentro Internacional sobre el Aprendizaje Significativo. [En línea]. Disponible en: http://www.if.ufrgs.br/ Moreira/apsigsubesp.pdf.

[6] Congreso de Colombia. Ley 1341 de 2009.

[7] OSILAC, El estado de las estadísticas sobre Sociedad de la Información en los Institutos Nacionales de Estadística de América Latina y el Caribe. Santiago de Chile: Medición de la Sociedad de la Información en América Latina y el Caribe, 2004. [En línea]. Disponible en: http://www. itu.int/wsis/stocktaking/docs/activities/1102712635/statistics-es.pdf. Fecha de consulta: 19 de febrero de 2014.

[8] J. Salinas Ibáñez, "Cambios metodológicos con las TIC: estrategias didácticas y entornos virtuales de enseñanza-aprendizaje," Bordón. Rev. Pedagog., vol. 56, no. 3, 2004, pp. 469-481.

[9] Ministerio de Educación, Competencias TIC para el desarrollo profesoral docente, Colombia aprende, 2013

[10] P. Marqués Graells, "Impacto de las Tic en la educación: Funciones y limitaciones," Cuad. Desarro. Apl. a las TIC, vol. 2, no. 1, 2013, p. 2.

[11] M. Cebrián y A. G. Rojas, Enseñanza virtual para la innovación universitaria, Madrid: Narcea Ediciones, 2003, pp. $22-29$.

[12] R. Carneiro, Los desafíos de las TIC para el cambio educativo. Madrid, España: Fundación Santillana, pp. 61-70.

[13] C. Sales Arasa, "Análisis de las estrategias de enseñanza con tecnologías de la información. ¿Un nuevo contexto metodológico de secundaria?," tesis de doctorado, Dept. Ped., Univ. València, Valencia, 2006.

[14] W. Cevallos, "Desarrollo de un marco referencial para la implantación, transferencia y evaluación de las TIC en las universidades," Tesis de maestría, Dept. Elec., and Comp, Esc. Sup. Pol. Lit, Guayaquil, Ecuador, 2008.

[15] J. Salinas Ibáñez, "Modelos flexibles como respuesta de las universidades a la sociedad de la información," Acción Pedagógica, vol. 11, no. 1, 2002, pp. 4-13.

[16] J. Carmargo, "Incorporación de las TIC en el aula de matemáticas en la básica primaria del centro educativo minas de Iracal sede la Honda," Pueblo Bello, 2010. [En línea]. Disponible en: http://www.eduteka.org/proyectos. $\mathrm{php} / 2 / 15053$.

[17] T. Rojano, "Incorporación de entornos tecnológicos de aprendizaje a la cultura escolar: Proyecto de innovación educativa en matemáticas y ciencias en escuelas secundarias públicas de México," Rev. Iberoam. Educ., no. 33, 2003, pp. 135-165.

[18] W. Gómez. "Significado que le dan los profesores al uso de las TIC en los procesos de enseñanza y de aprendizaje en dos instituciones educativas de Floridablanca". Tesis de doctorado, Dep. Ped., Univ. UIS, Santander, Colombia, 2008.

[19] J. Cabero Almenara, Nuevas tecnologías aplicadas a la educación. España: Mcgraw Hill, 2007, pp. 81-89.

[20] G. B. Pérez, F. B. Sáiz yA. F. i Miravalles, Didáctica universitaria en entornos virtuales de enseñanza-aprendizaje. España: Narcea Ediciones, 2006, pp. 34-41.

[21] G. Ramírez, Evaluación de introducción de Internet de objetos en espacios de aprendizaje. Tesis de doctorado, Dep. Ing. Telem., Univ. Carlos III Madrid, Madrid, España, 2010.

[22] Ó. H. Velásquez Arboleda, "El nuevo rol del docente virtual para entornos virtuales de aprendizaje, 'El caso CEIPA," Lupa Empres., no. 01, 2014. 
[23] R. Estrella, "Implicaciones pedagógicas y actitud del docente ante el uso de las TIC en el aula de ELE," 2013.

[24] G. Rodríguez, Utilización de objetos de aprendizaje para la enseñanza de la materia de tecnologías en la escuela secundaria, 2012. [En línea]. Disponible en: http://ilan.com.mx/wpcontent/uploads/2012/10/RedILAN.pdf. pp. 60-67.

[25] S. Molina Martín, "Una manera de integrar las herramientas tecnológicas en la formación de maestros," Rev. Electrónica Interuniv. Form. del Profr., vol. 12, no. 1, 2009, pp. 139-149.

[26] A. R. Lizcano Dallos y V. A. Uribe Otálora, "Corbaraque: Propuesta de un modelo para el desarrollo de Mec orientados a programas de educación a distancia," in VII Congreso Iberoamericano de Informática Educativa, 2004 , pp. 246-256.

[27] J. Beltrán Llera, Procesos, Estrategias y Técnicas de Aprendizaje. Madrid: Síntesis, 1993, pp. 53-60.

[28] J. De Zubiría, Los Modelos Pedagógicos, hacia una pedagogía dialogante. Instituto Alberto Merani, Bogotá, Colombia: Magisterio Editorial, 2006, pp. 15-18.

[29] D. J. Dorado, "Breve reflexión sobre entornos virtuales de aprendizaje," en XIII Encuentro de la Escuela Regional de Matemáticas, 2006.

[30] R. Blanco, A. Astorga, C. Guadalupe, R. Hevia, M. Nieto, M. Robalino y A. Rojas, "Educación de calidad para todos: un asunto de derechos humanos," en II Reunión Intergubernamental del Proyecto Regional de Educación para América Latina y el Caribe, 2007.

[31] Z. Sánchez y C. Castiglioni. "Educación y nuevas tecnologías”, España: Editorial Edunexo, 2000, pp. 26-31.

[32] Ministerio de Educación Nacional, "Definición Objeto Virtual de Aprendizaje," 2005. [En línea]. Disponible en: http://www.colombiaaprende.edu.co/html/directivos/1598/article-172369.html.

[33] I. I. Sánchez Medina, "Estado del arte de las metodologías y modelos de los Objetos Virtuales de Aprendizaje (OVAS) en Colombia," Rev. Entornos, vol. 2, no. 28, Apr. 2015, pp. 93-107.

[34] M. E. del Moral y D. A. Cernea, "Diseñando Objetos de Aprendizaje como facilitadores de la construcción del conocimiento," Presentado en II Simp. Pluridisciplinar sobre diseño, eval. y descr. de conte. Educ Reutilizables (SPDECE05.), 2007

[35] S. Nogales y V. Francés. Estrategias educativas, 2002 [En línea]. Disponible en: http://www.quadernsdigitals.net/datos_web/biblioteca/l_1343/enLinea/1.htm.

[36] D. Carbonell, "El concepto de la uve heurística," 2013. [En línea]. Disponible en: http://diegooctaviocarbonell9.blogspot.com.co/2013/02/el-concepto-de-la-uveheuristica.html.

[37] C. P. Mejía y J. A. Grisales, "Los estilos de aprendizaje y el tiempo empleado por el maestro dentro del aula de clase en la conducta del niño de preescolar y básica primaria de la Escuela Normal Superior Rebeca Sierra Cardona en el año 2012," 2012.
[38] Educarchile, "Conoce tu estilo de aprendizaje y estudia mejor.” [En línea]. Disponible en: http://www.educarchile. $\mathrm{cl} / \mathrm{ech} / \mathrm{pro} / \mathrm{app} / \mathrm{detalle}$ ID $=78032$

[39] Canales de Aprendizaje, Club de ensayos. [En línea]. Disponible en: https://www.clubensayos.com/ Psicolog\%C3\%ADa/Canales-De-Aprendizaje/243414. html

[40] A. H. Galvis Panqueva, Material educativo computarizado (MEC). Metodologías de desarrollo de software educativo. Santa Fe de Bogotá: Ediciones Uniandes, 1996, pp. 15-20.

[41] M. C. Leguizamón, Diseño y desarrollo de materiales educativos computarizados (MEC): una posibilidad para integrar la informática con las demás áreas del currículo, en VIII congreso de informática educativa Aprendizaje y currículo. [En línea]. Disponible en: http://revistavirtual.ucn.edu.co/index.php/RevistaUCN/article/ viewFile/190/365.

[42] Universidad Autónoma de México, Enseñanza a través de la computadora, 2008. [En línea]. Disponible en http://132.248.9.195/pd2006/0605150/A9.pdf.

[43] D. Cardona Román, C. Velásquez y V. Martínez, Desarrollo de un objeto virtual de aprendizaje como apoyo al curso mercadeo estratégico de la universidad nacional abierta y a distancia. Academia, 2015 [En línea]. Disponible en: http://www.academia.edu/3220544/ DESARROLLO_DE_UN_OBJETO_VIRTUAL_DE APRENDIZAJE_COMO_APOYO_AL_CURSO_MERCADEO_ESTRAT\%C3\%89GICO_DE_LA_UNIVERSIDAD_NACIONAL_.

[44] R. Zatarain Cabada y M. L. Barrón Estrada, "Herramienta de autor para la identificación de estilos de aprendizaje utilizando mapas auto-organizados en dispositivos móviles," Rev. Electrónica Investig. Educ., vol. 13, no. 1, mayo 2011.

[45] H. Fontalvo, F. Iriarte, E. Domínguez, C. Ricardo, B. Ballesteros, V. Muñoz, y J. Campo, "Diseño de ambientes virtuales de enseñanza-aprendizaje y sistemas hipermedia adaptativos basados en modelos de estilos de aprendizaje," Zo. Próxima, 2007, pp. 42-61.

[46] Universidad de Alicante, "Modelo vista controlador (MVC).” [En línea]. Disponible en: http://si.ua.es/es/documentacion/asp-net-mvc-3/1-dia/modelo-vista-controladormvc.html.

[47] "Flex - Tutoriales de Flex, videotutoriales y artículos." [En línea]. Disponible en: http://www.cristalab.com/tags/ flex/.

[48] "Adobe Flash." [En línea]. Disponible en: http://www. puntocad.com/index.php/component/jshopping/product/ view/11/26? Itemid=0.

[49] C. Lunar, "Aprender lenguaje de programación PHP." [En línea]. Disponible en: http://lunarwarinftec.blogspot.com. co/2015/03/aprender-lenguaje-de-programacion-php.html.

[50] E. Toledo, ¿Qué es MySQL? [En línea]. Disponible en: http://www.gridmorelos.uaem.mx/ mcruz//cursos/miic/ MySQL.pdf. 